JOURNAL OF SYNCHROTRON RADIATION

ISSN 1600-5775

Received 14 August 2019

Accepted 19 February 2020

Edited by R. W. Strange, University of Essex, UK

Keywords: QEXAFS; fluorescence XAS; perovskite-type oxides.

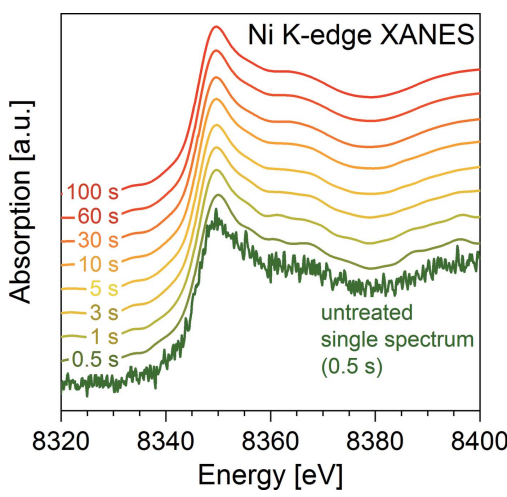

C 2020 International Union of Crystallography

\section{Fluorescence-detected quick-scanning X-ray absorption spectroscopy}

\author{
Adam H. Clark, ${ }^{a}$ Patrick Steiger, ${ }^{a}$ Benjamin Bornmann, ${ }^{\text {b }}$ Stephan Hitz, \\ Ronald Frahm, ${ }^{\mathrm{b}}$ Davide Ferri $^{\mathrm{a}}$ and Maarten Nachtegaal ${ }^{\mathrm{a} *}$

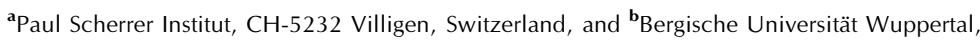 \\ D-42119 Wuppertal, Germany. *Correspondence e-mail: maarten.nachtegaal@psi.ch
}

Time-resolved X-ray absorption spectroscopy (XAS) offers the possibility to monitor the state of materials during chemical reactions. While this technique has been established for transmission measurements for a number of years, XAS measurements in fluorescence mode are challenging because of limitations in signal collection as well as detectors. Nevertheless, measurements in fluorescence mode are often the only option to study complex materials containing heavy matrices or in samples where the element of interest is in low concentration. Here, it has been demonstrated that high-quality quick-scanning full extended X-ray absorption fine-structure data can be readily obtained with sub-second time resolution in fluorescence mode, even for highly diluted samples. It has also been demonstrated that in challenging samples, where transmission measurements are not feasible, quick fluorescence can yield significant insight in reaction kinetics. By studying the fast high-temperature oxidation of a reduced $\mathrm{LaFe}_{0.8} \mathrm{Ni}_{0.8} \mathrm{O}_{3}$ perovskite type, an example where the perovskite matrix elements prevent measurements in fluorescence, it is shown that it is now possible to follow the state of $\mathrm{Ni}$ in situ at a $3 \mathrm{~s}$ time resolution.

\section{Introduction}

X-ray absorption spectroscopy (XAS) is a potent tool to study the oxidation state and local coordination environment of heterogeneous catalysts during operation (operando methodology) (Nachtegaal et al., 2017). When XAS is achieved with good time resolution ( $10 \mathrm{~s}$ or better) it allows for the determination of the structure of reactive species in transient experiments (Marberger et al., 2018). Both energy-dispersive XAS (Nagai et al., 2008; Pascarelli et al., 1999) and quickscanning extended X-ray absorption fine-structure (QEXAFS) spectroscopy allow the collection of full EXAFS spectra in transmission mode with sub-second time resolution (Frahm, 1988; Fonda et al., 2012; Müller et al., 2016; Lützenkirchen-Hecht et al., 2001). In previous years, QEXAFS has been mainly exploited for the collection of transmission spectra using gas-ionization chamber detectors, as these offer excellent linearity and simple adjustability of the signal intensity (Nachtegaal et al., 2015). Furthermore, the introduction of gridded ionization chambers allowed one to significantly increase the response time of the ionization chambers and thus the time resolution of XAS (Müller et al., 2013). There exist cases where the collection of time-resolved spectra is desirable but the material of interest does not allow the acquisition of transmission spectra. Materials with a low concentration of the element of interest and/or the presence of heavy elements, which absorb very strongly in the selected energy range, result in transmission XAS measurements not being feasible. In this case, fluorescence measurements are 
desirable but present additional challenges. Since the fluorescence radiation is emitted isotropically, a large detector area is necessary to achieve high efficiency.

Multi-element solid-state detectors (SSDs), which are commonly used for fluorescence XAS measurements, suffer from low saturation count rates and low response times, which typically limit their application in time-resolved studies compared with gas-ionization chambers (Nachtegaal et al., 2015). SSDs are typically limited to a count rate of several $\mathrm{MHz}$ when operating in photon-counting mode with modern readout electronics. For recording QEXAFS spectra, with $1 \mathrm{MHz}$ counting rate from an SSD, with up to ten spectra per second and 1000 data points each, each data point would be acquired in $\sim 100 \mu$ s or 100 counts per point at maximum count rate, which gives a too large statistical error of $10 \%$ (based on the Poisson limit, signal-to-noise ratio $\simeq N^{0.5}$, where $N$ is the number of samples at each data point). In order to achieve acceptable data quality, reducing the statistical error to $1 \%$, count rates well in excess of $100 \mathrm{MHz}$ would be required (Hansen et al., 2008). Typically, experiments performed on all but very dilute samples yield a fluorescence flux in excess of $10^{6}$ photons $\mathrm{s}^{-1}$, which causes saturation in a SSD detector. Alternatively, avalanche photodiodes (APDs) with maximum count rates of $\sim 100 \mathrm{MHz}$ could be used. Such systems are often used in pump-probe XAS experiments (Smolentsev et al., 2018; Wang et al., 2017). However, in contrast to large-area partially depleted passivated implanted planar silicon (PIPS) detectors, typically both SSDs and APDs are limited by the area of the detectors and as such the measureable solid angle of the fluorescence emitted from a sample.

Previously it has been shown that high-quality X-ray absorption near-edge structure (XANES) spectra can be recorded on a sub-second time scale using fluorescencedetected QEXAFS (Lützenkirchen-Hecht et al., 2001). Elsewhere, using a highly reversible system, the application of a modulation approach to recover time resolution through repeatedly cycling the experiment at different incident X-ray energies allowed for the reconstruction of the XANES spectra with a moderate time resolution (Guda et al., 2018). However, such an approach requires significant time to perform a single experiment and a full reproducibility of the chemical process. There are many cases, however, where such a time resolution is potentially of great value to enable the observation of fast irreversible processes.

Alternative methods have been employed to gain time resolution with fluorescence measurements. For instance, Turbo-XAS allows for the collection of spectra in fluorescence mode at second time resolution at energy-dispersive beamlines by scanning a slit along the energy axis of the detector (Pascarelli et al., 1999). By using Turbo-XAS the redispersion of $\mathrm{Pt}$ on a ceria-zirconia support has been elucidated with moderate time resolution achieved for reasonable quality XANES spectra (6 s) (Nagai et al., 2008). However, the TurboXAS method has its limitations. In particular, the limited energy bandwidth resulting from the Bragg angle variation along the incident-beam footprint on the polychromator crystal. As a consequence, the total energy range measureable is significantly reduced at low energies (Abe et al., 2018). This limitation typically makes full EXAFS data acquisition using Turbo-XAS infeasible at energies lower than $\sim 7 \mathrm{keV}$. Additionally, the change in direction of the slit motion results in significant dead-time between acquisitions, $\sim 800 \mathrm{~ms}$ (Pascarelli et al., 1999). Furthermore, the advent of new-generation lower-emittance storage rings brings significant new challenges to energy-dispersive XAS beamlines. As a consequence of the XAS spectrum being acquired as a one-dimensional image, the larger horizontal coherence length and its negative effects on the spatial homogeneity of the beam will affect energy-dispersive XAS more than any other XAS method (Abe et al., 2018).

Another method for measuring fluorescence XAS spectra with high time resolution is by means of high energy resolved off-resonant spectroscopy (HEROS) using highly resolving X-ray emission spectrometers (Szlachetko et al., 2012; Błachucki et al., 2014). In this case, an XAS-like spectrum is collected at one incident energy, below the resonant region of the absorption edge of interest. A dispersive emission spectrometer then allows collecting the high energy resolved XAS spectrum in a single shot (Marchionni et al., 2016). However, since the X-ray cross section becomes small below the absorption edge, and the solid angle of the X-ray emission spectrometer is typically limited, this method is not applicable for dilute samples. Moreover, HEROS cannot probe the EXAFS region. Nevertheless, it is an excellent method to collect fluorescence XAS spectra free of self-absorption (Błachucki et al., 2014).

Here we present the current state of fluorescence-detected XAS spectra, as can be collected at the SuperXAS beamline using a QEXAFS monochromator, with the best time resolution presented to date for the study of both dilute samples or elements in samples with a heavily absorbing matrix.

\section{Results and discussion}

For fluorescence-detected time-resolved quick-scanning XAS measurements reported herein, a PIPS fluorescence detector with an active diameter of $39 \mathrm{~mm}$ and a thickness of $300 \mu \mathrm{m}$ from Mirion Technologies has been utilized. No bias potential was applied and the capacitance of the PIPS detector used is $294 \mathrm{pF}$. The current yield from the PIPS is amplified using a very simple amplifier consisting only of the LMP7721 integrated circuit, together with a corresponding gain resistor and a tiny capacitor to limit the internal bandwidth of the chip, Fig. 1(a). The so-called trans-impedance amplifier is battery powered $( \pm 4.5 \mathrm{~V})$ to reach the best possible signal-to-noise ratio. The internal capacitance of the PIPS detector introduces several instabilities like ringing, showing in spectra as additional oscillations behind the edge. To suppress such effects, it is therefore necessary to use a feedback capacitor. The drawback of increasing feedback capacitance results in the rise time of the amplifier chain being limited to several microseconds. 


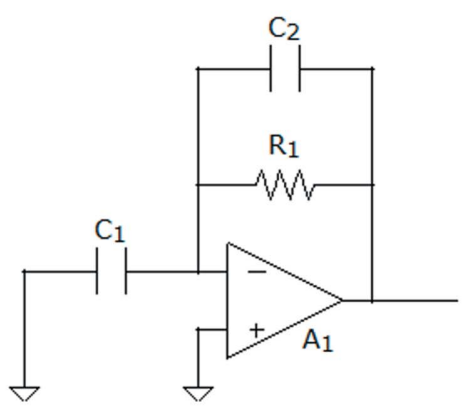

(a)

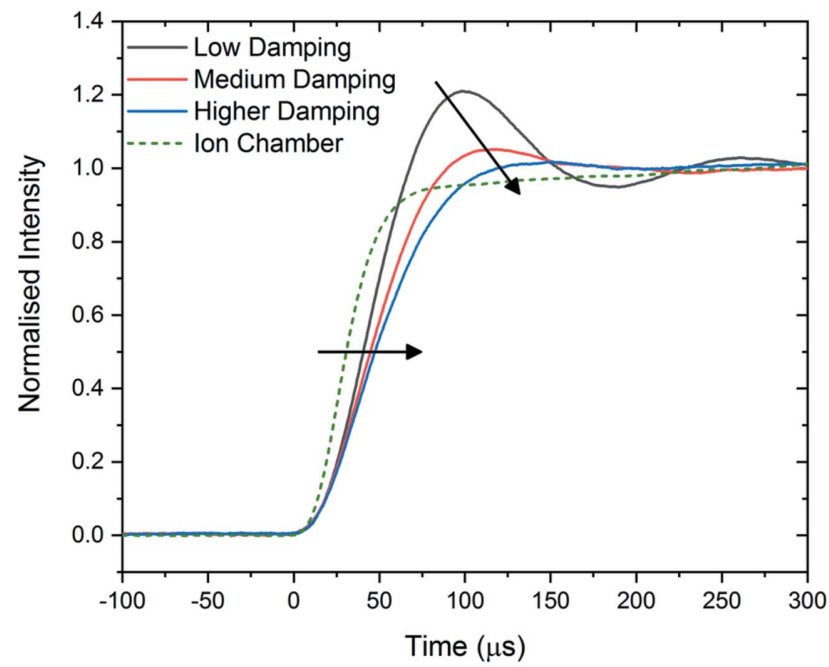

(b)

Figure 1

(a) Circuitry diagram for the amplifier. $\mathrm{C} 1$ describes the capacitance of the PIPS detector, $\mathrm{C} 2$ describes the feedback capacitance, R1 describes the gain resistor and A1 describes the trans-impedence amplifier. (b) The effect of increasing the feedback capacitance on the step response and rise time of the amplifier utilized and comparison with the gridded ion chamber and Keithley amplifier.

To optimize the feedback capacitance and as such reduce artefacts in measurements recorded with fast scanning through an absorption edge, the step response of the amplified PIPS signal was measured using a fast X-ray chopper rotating at $200 \mathrm{~Hz}$ at the SuperXAS beamline. An idealized $13 \mathrm{~mm}$ diameter $\mathrm{NiO}$ pellet (transmission edge step of 1) was mounted after the X-ray chopper and before the second ionization chamber at $90^{\circ}$ to the incident X-ray beam. The PIPS detector was placed at $\sim 30^{\circ}$ angle from the incident beam and facing the $\mathrm{NiO}$ pellet. The incident beam was tuned to $8.4 \mathrm{keV}$ to yield fluorescence emission from the $\mathrm{NiO}$ pellet. The measurement data were recorded using a National Instruments PXIe-6366 multifunctional data-acquisition board (DAQ). The detector currents from either ion chambers or the PIPS fluorescence detector are amplified and the resulting voltages are digitized by analogue-to-digital converters of the DAQ with 16 bit resolution and sampled at $2 \mathrm{MHz}$.

The step response of the PIPS diode to incoming X-rays has a negligible rise time for QEXAFS applications but is followed by strong oscillations for several hundred microseconds when insufficiently damped. The influence of the damping with increasing feedback capacitance is demonstrated in Fig. 1(b). Ringing of the step response is observed when insufficient feedback capacitance is used. However, increasing the feedback capacitance, whilst alleviating the ringing of the step response to the incident fluorescence, results in a slight increase in the rise time of the amplifier.

Using the optimized amplifier feedback capacitance, the sensitivity and data-collection repetition rates were explored to determine the potential useable operation limits. Three dilutions of $\mathrm{NiO}$ were prepared and pressed into $13 \mathrm{~mm}$ pellets. The mass of $\mathrm{NiO}$ within each pellet was estimated by consideration of the transmission XAS edge step obtained. Transmission edge steps of $0.120,0.018$ and 0.008 were observed from $\sim 0.7,0.1$ and $0.05 \mathrm{mg}$ of $\mathrm{NiO}$ diluted in cellulose. The total mass of each pellet was measured as $\sim 50 \mathrm{mg}$. The quick-scanning fluorescence and transmission XAS data were processed using ProQEXAFS (Clark et al., 2020) and utilized third-order Butterworth filtering for high-frequency noise suppression. Significant signal-to-noise ratio improvements, approximately by a factor of four, are readily achievable using Butterworth filtering applied to highly oversampled XAS data (Clark et al., 2020). The subsequently obtained normalized quick-scanning fluorescence XAS spectrum for each sample with a data-acquisition time of $1 \mathrm{~s}$ (averaging both the up and down directions) at a monochromator oscillation repetition rate of $1 \mathrm{~Hz}$ are shown in Fig. 2(a). Comparison between the XAS data collected in transmission geometry and in fluorescence geometry is given in Fig. 2(b) demonstrating the fluorescence XAS quality and agreement between the two measurement modes for $1 \mathrm{~s}$ data-acquisition periods.

The data quality achievable on such low concentrations of $\mathrm{NiO}$, and in short acquisition periods (1 s), shows the versatility of the PIPS detector for time-resolved fluorescence detection XAS. Comparison of the XANES obtained at $1 \mathrm{~Hz}$ monochromator oscillation for the most dilute sample (transmission edge step of 0.008 ) for $60 \mathrm{~s}$ averaged spectrum and $1 \mathrm{~s}$ (averaged in the up and down motion of the monochromator) data-acquisition period obtained in total fluorescence yield and with $1 \mathrm{~s}$ in transmission geometry are shown in Fig. 3(a). It is evident that for $1 \mathrm{~s}$ acquisition time the data obtained in fluorescence is of a higher quality compared with the transmission data and is similar to the $60 \mathrm{~s}$ averaged spectra. The extraction of the EXAFS in $k$ space and $R$ space is given in Figs. $3(b)$ and $3(c)$, respectively. The Fourier transform window used was between 2.5 and $10.2 \AA^{-1}$. From these figures, it is further evident that the transmission signal has a significantly increased noise level compared with what is achieved in total fluorescence yield with the PIPS detector. The transmission signal shows a greatly increased noise level in $k$ space and yields an $R$-space pattern with significant artefacts as a result. In contrast, the $1 \mathrm{~s}$ and $60 \mathrm{~s}$ data acquisition in fluorescence demonstrate very similar profiles in both $k$ space and $R$ space. As such it can be noted that the $1 \mathrm{~s}$ data acquisition, even in the case of a very dilute sample, is not limiting the quality of the data obtainable from this sample using the PIPS detector. 


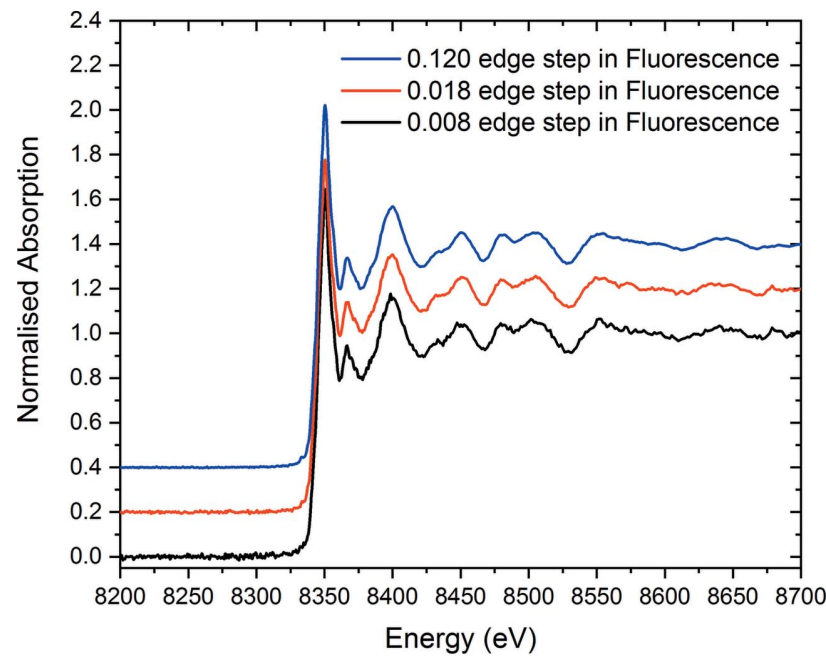

(a)

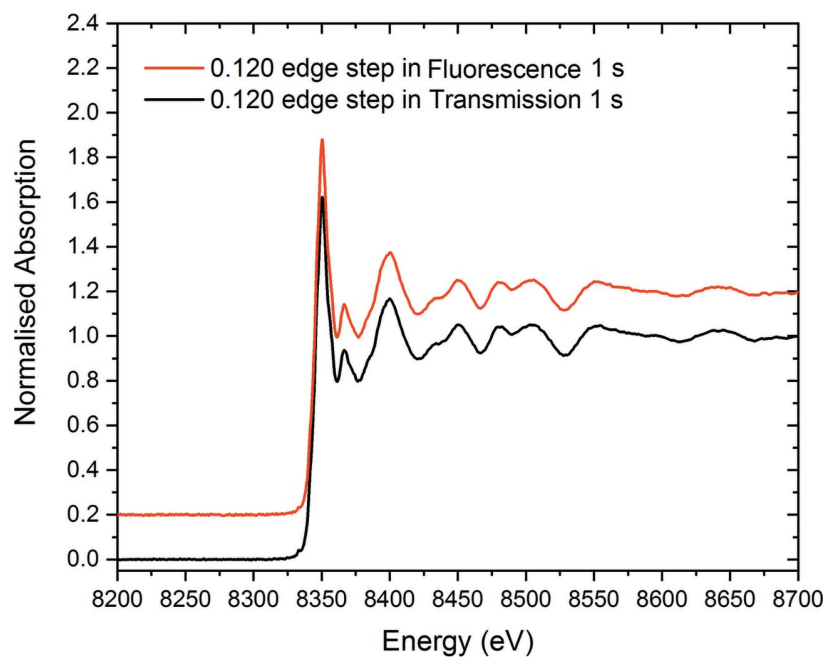

Figure 2

(b)

(a) Normalized Ni $K$-edge $(8333 \mathrm{eV})$ XAS spectra measured in the fluorescence of $\mathrm{NiO}$ samples with decreasing sample mass pressed into $13 \mathrm{~mm}$ pellets. The absorption-edge step from each sample was measured from the transmission geometry data. (b) Comparison of the normalized XAS spectra obtained in $1 \mathrm{~s}$ for the 0.120 edge-step NiO pellet obtained in transmission and fluorescence modes.

To explore the effect of the data-acquisition period on the quality of the data obtainable in fluorescence detection, increasing monochromator oscillation frequency from $1 \mathrm{~Hz}$ to $10 \mathrm{~Hz}$ has been undertaken. Fig. 4(a) gives the XANES region of the very dilute $\mathrm{NiO}$ sample with increasing monochromator oscillation frequency. The data plotted are the average of $60 \mathrm{~s}$ data acquisition at each oscillation frequency. With increasing monochromator oscillation frequency, slight distortions in the XANES region are observed. Higher monochromator oscillation frequencies lead to a smoothing effect as a result of the finite rise time of the amplifier used. As such using the PIPS detector with monochromator oscillation frequencies in excess of $1 \mathrm{~Hz}$ should be carried out with care. However, for following trends in oxidation-state changes and speciation changes during reactive chemistry, the resolution on the XANES is still sufficient even at $10 \mathrm{~Hz}$. Fig. 4(b) gives the

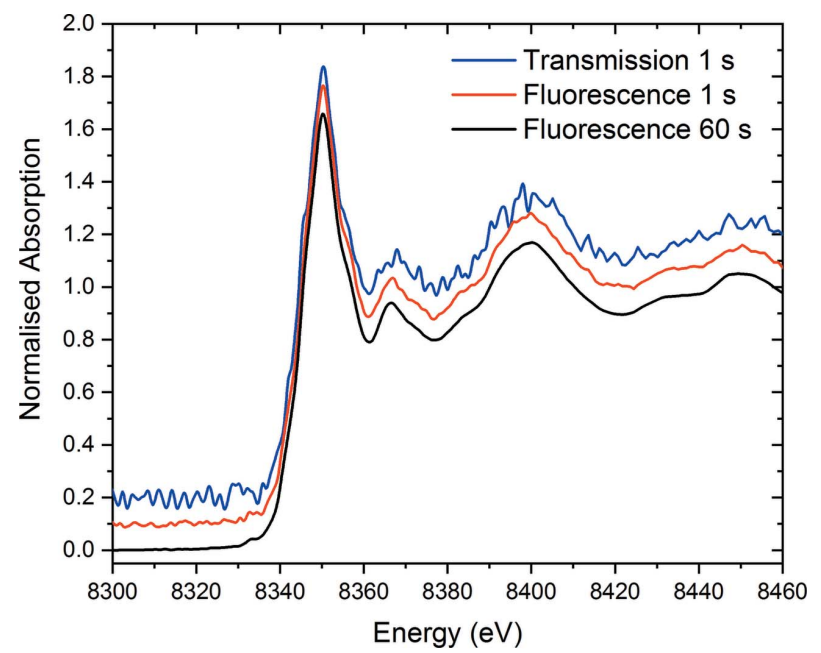

(a)

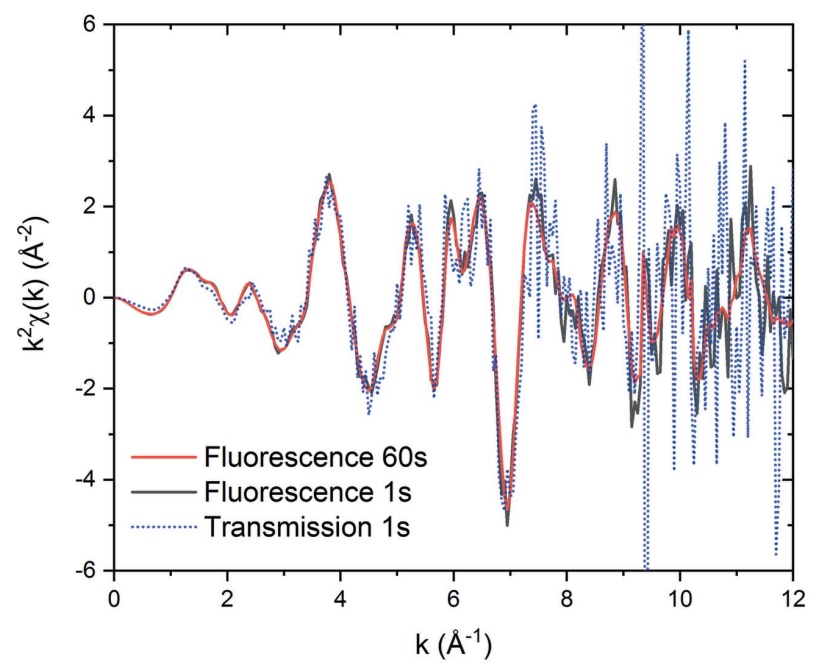

(b)

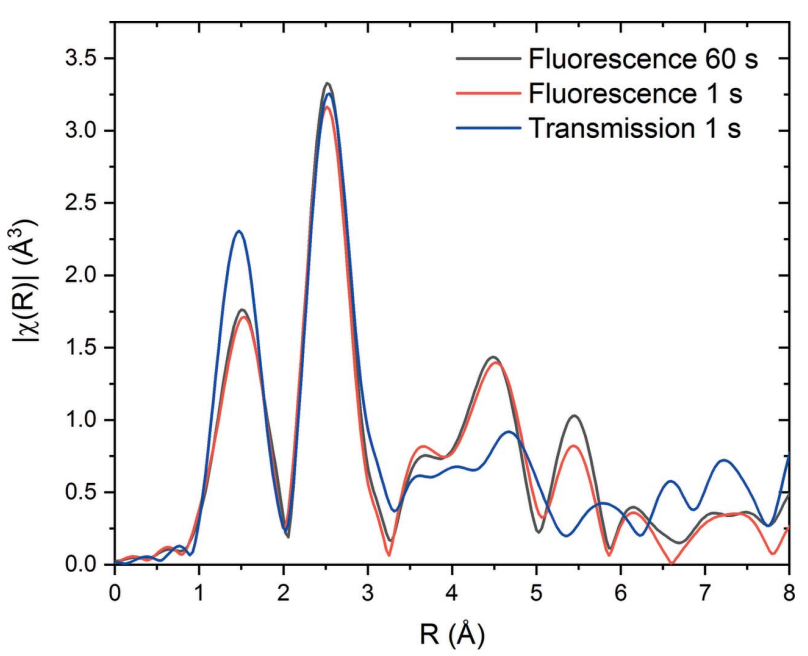

(c)

Figure 3

Normalized Ni $K$ edge ( $8333 \mathrm{eV}$ ) XAS data of a very dilute NiO sample comparing the $1 \mathrm{~s}$ transmission (0.008 edge step), $1 \mathrm{~s}$ fluorescence detection and $60 \mathrm{~s}$ averaged fluorescence in blue, red and black, respectively. The XANES region, $k^{2}$-weighted $k$ space and $R$-space spectra are shown in $(a),(b)$ and $(c)$, respectively. 


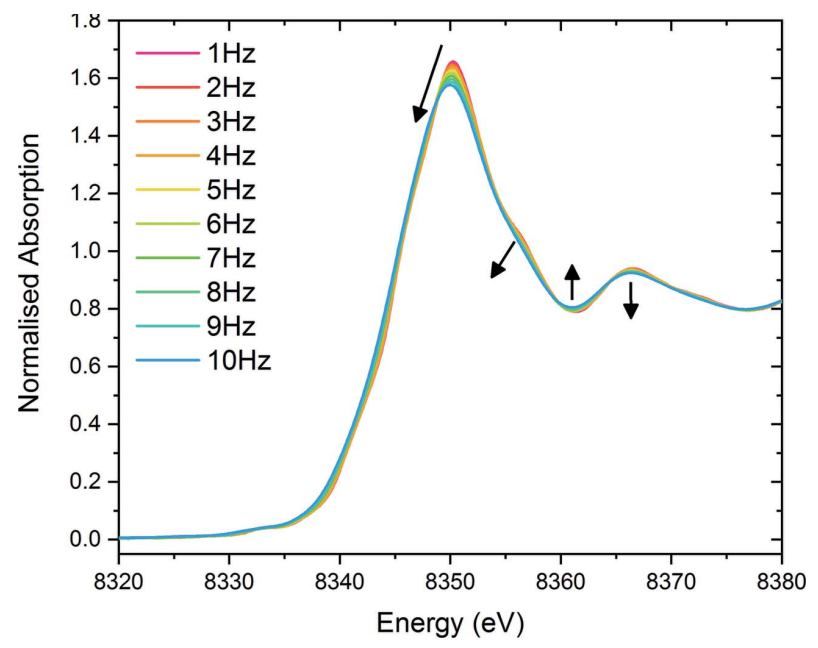

(a)

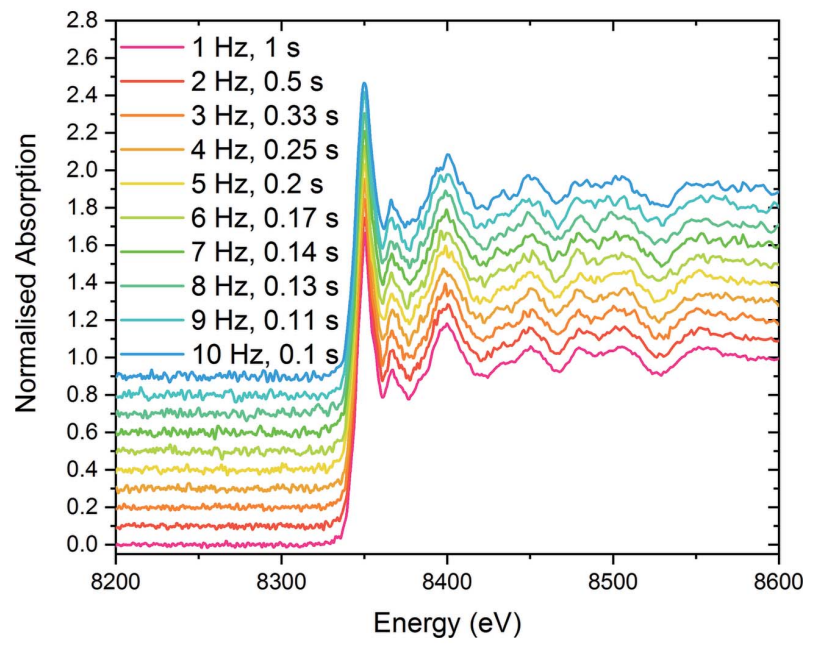

(b)

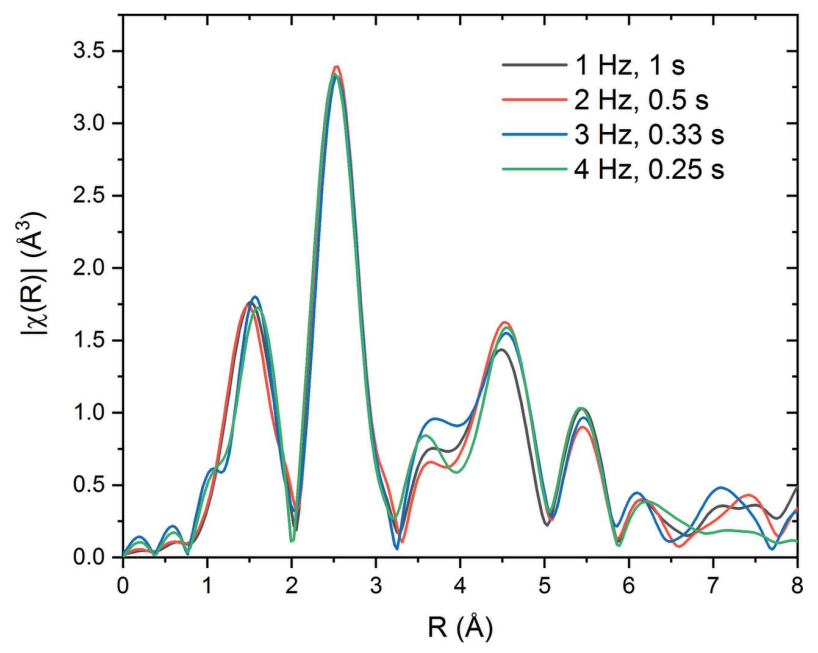

(c)

Figure 4

(a) Normalized $\mathrm{Ni} K$-edge $(8333 \mathrm{eV})$ XANES data measured in fluorescence of a very dilute $\mathrm{NiO}$ sample (0.008 edge step), comparing a $60 \mathrm{~s}$ averaged data-acquisition period for increasing monochromator oscillation frequency. (b) A stacked plot of the extended XAS data collected with increasing monochromator oscillation frequency and decreased total signal-acquisition period. (c) Obtained $R$-space data of 1 to $0.25 \mathrm{~s}$ data-acquisition periods. average of the up and down spectra acquired at each monochromator oscillation frequency corresponding to 1 to $0.1 \mathrm{~s}$ data-acquisition periods from 1 to $10 \mathrm{~Hz}$. Here it is demonstrated that even at very short data-acquisition periods the quality of the obtainable data is still outstanding considering the high dilution (transmission edge step of 0.008) of the $\mathrm{NiO}$ sample. Whilst transmission data could not yield high enough quality for the EXAFS region on this sample, useable quality EXAFS data are obtained from the PIPS detector with even $4 \mathrm{~Hz}$ monochromator oscillation frequency $(0.25 \mathrm{~s}$ data acquisition), Fig. 4(c). The noise level at frequencies higher than $4 \mathrm{~Hz}$ did not allow for the Fourier transformation to $R$ space between 2.5 and $10.2 \AA^{-1}$ to give reliable data from the very dilute $\mathrm{NiO}$ sample investigated (0.008 edge step).

Being able to measure time-resolved XAS spectra in fluorescence mode has obvious advantages. For the study of dilute materials, where transmission XAS no longer yields useable data, we have demonstrated that fluorescence XAS spectra can be acquired with $0.1 \mathrm{~s}$ time resolution for XANES and $0.25 \mathrm{~s}$ for EXAFS. Another class of materials which are otherwise not measureable in transmission can also significantly benefit from quick fluorescence data acquisition. One example involves the study of perovskite-type oxides $\left(\mathrm{ABO}_{3}\right)$ (Peña \& Fierro, 2001) where obtaining XAS of dopant elements substituting the B-site transition metal is often challenging. The heavy A-site element (lanthanide, alkali or Earth alkali element) typically absorbs very strongly at the lower energies needed to probe the local environment of the catalytically active B-site elements. As such, transmission measurements become infeasible because of the total absorption of the X-ray beam by the sample with a typical quantity of material used in operando XAS experiments.

Perovskites have attracted great attention in recent years for their application as active materials in electrochemical cells (e.g. electrolyzers, solid oxide fuel cells) (Sapountzi et al., 2017; Jiang, 2008; Neagu et al., 2015; Fabbri et al., 2017). They have also received attention in classical heterogeneous catalysis in part because of their ability to reversibly segregate their B-site elements to produce active metal nanoparticles at the oxide surface during reduction and reincorporate these elements back into their structure during reoxidation (Nishihata et al., 2002; Steiger et al., 2017; Onn et al., 2018). This socalled structural reversibility allows for the generation of redox stable and regenerable metal catalysts (Steiger et al., 2018). Here we demonstrate the combination of fluorescence QEXAFS, which enables the speciation of $\mathrm{Ni}$ in $\mathrm{LaFe}_{0.8} \mathrm{Ni}_{0.2} \mathrm{O}_{3}(4.8 \mathrm{wt} \% \mathrm{Ni})$ using fluorescence QEXAFS at the Ni $K$ edge $(8333 \mathrm{eV})$, and the in situ observation of $\mathrm{Ni}$ reincorporation during reoxidation at $650^{\circ} \mathrm{C}$ at a time resolution of $3 \mathrm{~s}$.

Fig. 5 displays XANES spectra of $\mathrm{LaFe}_{0.8} \mathrm{Ni}_{0.2} \mathrm{O}_{3}$ collected around the Ni $K$ edge $(8333 \mathrm{eV})$ in fluorescence mode for a single spectrum and averaged data. Because of the strongly absorbing nature of the perovskite there is no transmission through the sample. The spectra were collected at $1 \mathrm{~Hz}$ and the fluorescence signal was recorded with a $2 \mathrm{MHz}$ sampling frequency and processed using ProQEXAFS (Clark et al., 


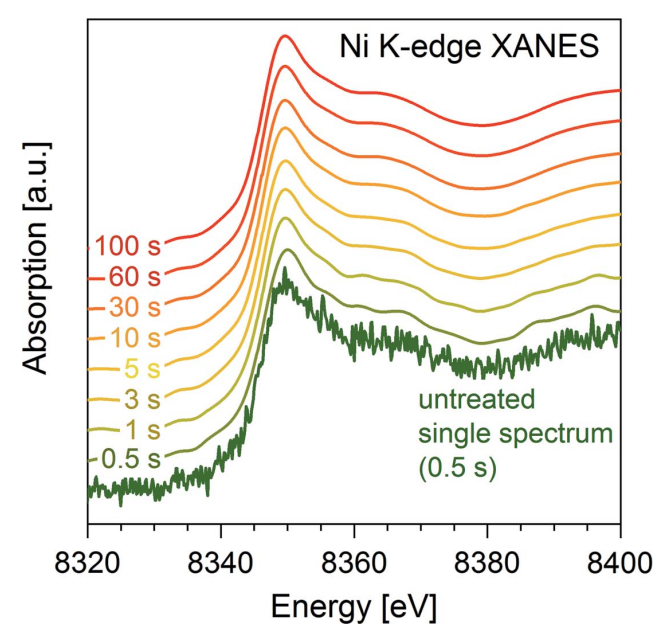

Figure 5

Normalized $\mathrm{Ni} K$-edge $(8333 \mathrm{eV})$ XANES spectra of the calcined $\mathrm{LaFe}_{0.8} \mathrm{Ni}_{0.2} \mathrm{O}_{3}$ perovskite-type oxide for different averaging times $(1-100 \mathrm{~s})$. The quality of a normalized single raw spectrum (untreated, $0.5 \mathrm{~s})$ and the same spectrum after noise filtering $(0.5 \mathrm{~s})$ are shown for comparison.

2020). Suppression of high-frequency noise was achieved using a third-order Butterworth filter in combination with a weak moving time-frame Savitzky-Golay filter. The Butterworth filter is a smooth and continuous low-pass filter allowing for the removal of high-frequency noise signals. Through this procedure it is possible to achieve a temporal resolution of at least $3 \mathrm{~s}$ for the XANES region. Averaging over longer times (10-100 s) did not further improve the quality of the XANES, as shown in Fig. 5. Therefore, we demonstrate that time resolutions in the second range can also be achieved on challenging materials, such as the perovskite-type oxides presented here, using the PIPS fluorescence detector and the continuous signal readout applied in this work. The time resolution achievable in this case is lower than what was possible in the dilute $\mathrm{NiO}$ pellet because of the significant background fluorescence from $\mathrm{La}$ and $\mathrm{Fe}$ within the perovskite sample.

The possibilities offered by using the appropriate instrumentation and data-processing methods were exploited to observe in situ the reincorporation of $\mathrm{Ni}$ metal back into the perovskite-type lattice of $\mathrm{LaFe}_{0.8} \mathrm{Ni}_{0.2} \mathrm{O}_{3}$ during reoxidation in $20 \mathrm{vol} \% \quad \mathrm{O}_{2} / \mathrm{N}_{2}$ at $650^{\circ} \mathrm{C}$. This process is known to occur in some pre-reduced perovskite-type oxides at sufficiently high temperatures. Furthermore, this process is known to be essential for the observed redox stability of the catalyst and can be exploited to oxidatively regenerate the catalyst from poisoning species (Steiger et al., 2018, 2017; Burnat et al., 2016). Prior to the experiment, $\mathrm{LaFe}_{0.8} \mathrm{Ni}_{0.2} \mathrm{O}_{3}$ was reduced ( 10 vol\% $\left.\mathrm{H}_{2} / \mathrm{Ar}, 600^{\circ} \mathrm{C}, 1 \mathrm{~h}\right)$, which is known to cause the preferential reduction and segregation of metallic $\mathrm{Ni}$ from $\mathrm{LaFe}_{0.8} \mathrm{Ni}_{0.2} \mathrm{O}_{3}$ leading to the formation of metallic Ni particles on the surface of the Nidepleted perovskite crystallites. With the methodological advancements presented in this work it is now possible to follow such transient changes in the state of $\mathrm{Ni}$ in situ at suitable time resolutions. Fig. 6(a) displays the results of $\mathrm{Ni}$ speciation by linear combination analysis using the spectra of $\mathrm{Ni}$ in the perovskite octahedral coordination of $\mathrm{LaFe}_{0.8} \mathrm{Ni}_{0.2} \mathrm{O}_{3}\left(\mathrm{Ni}_{\text {oct }}^{n+}, n>2\right)$ (Steiger et al., 2017) and of metallic $\mathrm{Ni}\left(\mathrm{Ni}^{0}\right)$ and $\mathrm{NiO}$ reference spectra [Fig. 6(b)]. Upon subjecting reduced $\mathrm{LaFe}_{0.8} \mathrm{Ni}_{0.2} \mathrm{O}_{3}$ to the oxidizing feed at $650^{\circ} \mathrm{C}, \mathrm{Ni}^{0}$ is oxidized simultaneously to $\mathrm{Ni}_{\text {oct }}^{n+}$ and $\mathrm{NiO}$ species in the first $10-15 \mathrm{~s}$. Within this time, $\mathrm{Ni}^{0}$ vanishes completely. The fraction of $\mathrm{NiO}$ passes through a maximum at $24 \mathrm{~s}$ before decreasing continuously, while the concentration of $\mathrm{Ni}_{\text {oct }}^{n+}$ species increases steadily. After $\sim 1 \mathrm{~h}$ all Ni has been reincorporated back into the $B$ site of the perovskite lattice. At this point, the octahedral coordination environment of $\mathrm{Ni}$ is identical to that obtained after synthesis and its absorption spectrum resembles that observed for $\mathrm{LaFe}_{0.8} \mathrm{Ni}_{0.2} \mathrm{O}_{3}$ [Fig. 6(b)]. Acquisition times of a single spectrum with previously applied methods of collecting the fluorescence signal using slow energy scanning and silicon drift detectors were in the range of $\sim 10 \mathrm{~min}$, which limited the observation of transient processes with faster kinetics significantly.

Further improvements can be made to the detection system by increasing the solid angle of the fluorescence detection, either by increasing the detector area or through the use of multiple detectors to enhance the collected fluorescence signal per time and therefore the potential time resolution. Nevertheless, these results demonstrate that it is possible with the current technology to achieve respectable time resolution through fluorescence detection in situ QEXAFS, and on challenging samples, and elucidate previously unresolvable time-dependent processes with application of the methodology presented in this work.

\section{Experimental}

$\mathrm{LaFe}_{0.8} \mathrm{Ni}_{0.2} \mathrm{O}_{3}$ was prepared via the amorphous citrate process (Marcilly et al., 1970) using $\mathrm{La}\left(\mathrm{NO}_{3}\right)_{3} \cdot 6 \mathrm{H}_{2} \mathrm{O}$ (Sigma-Aldrich, $99.999 \%$ trace-metals basis), $\mathrm{Fe}\left(\mathrm{NO}_{3}\right)_{3} \cdot 9 \mathrm{H}_{2} \mathrm{O}$ (Sigma-Aldrich, $\geq 99.95 \%$ trace-metals basis), $\mathrm{Ni}\left(\mathrm{NO}_{3}\right)_{2}$. $6 \mathrm{H}_{2} \mathrm{O}$ (Sigma-Aldrich, $99.999 \%$ trace-metals basis) and citric acid (Sigma-Aldrich, ACS reagent, $\geq 99.5 \%$ ). The precursor

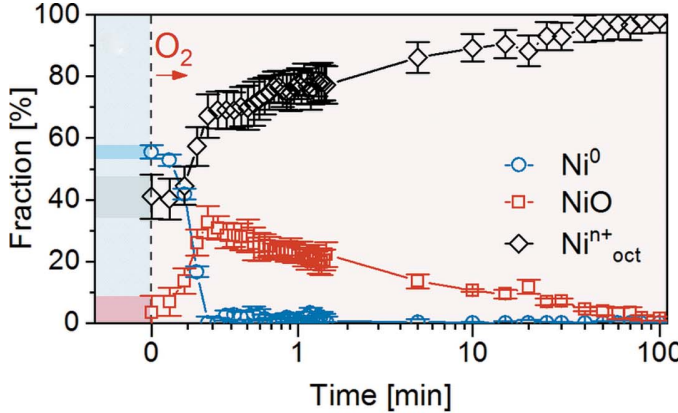

(a)

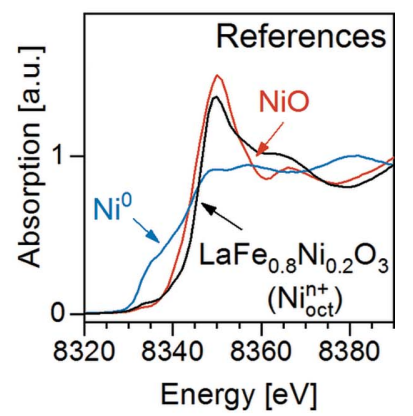

(b)

(a) Time-resolved in situ Ni speciation obtained through linear combination fitting during reoxidation of pre-reduced $\mathrm{LaFe}_{0.8} \mathrm{Ni}_{0.2} \mathrm{O}_{3}$ at $650^{\circ} \mathrm{C}$ in $20 \mathrm{vol} \% \mathrm{O}_{2} / \mathrm{Ar}$. (b) $\mathrm{Ni} K$-edge XANES spectra of reference components used for linear combination fitting. 
was calcined at $700^{\circ} \mathrm{C}$ for $2 \mathrm{~h}$ after drying $\left(70^{\circ} \mathrm{C}, 12 \mathrm{~h}\right)$ to obtain the perovskite-type mixed oxide. Extensive material characterization has already been reported elsewhere (Steiger et al., 2017, 2018). Ni $K$-edge $(8333 \mathrm{eV})$ XAS data were collected at beamline X10DA (SuperXAS) of the Swiss Light Source (SLS, Villigen, Switzerland). The beam energy and beam current within the storage ring were $2.4 \mathrm{GeV}$ and $400 \mathrm{~mA}$, respectively. XAS spectra were collected in fluorescence mode using an ionization chamber filled with $\mathrm{N}_{2}$ to measure incoming beam intensity and a PIPS detector mounted at $90^{\circ}$ to the incoming X-ray beam and the sample mounted at $45^{\circ}$. The beam was collimated using a Si-coated mirror, which was also used for the reduction of higher harmonic contributions while the X-ray energy was continuously scanned around the Ni $K$ edge using a Si (111) channelcut monochromator at a monochromator oscillation frequency of $1 \mathrm{~Hz}$ (Müller et al., 2016). The beam was focused to a spot size of $1.0 \mathrm{~mm} \times 0.2 \mathrm{~mm}$ (height $\times$ width) using an Rh-coated toroidal mirror. Measurements were performed on powder samples mounted inside a quartz capillary (Hilgenberg, outer diameter $3.0 \mathrm{~mm} / \mathrm{inner}$ diameter $2.9 \mathrm{~mm}$ ). Gas flow was controlled during in situ measurements using mass-flow controllers (Bronkhorst) and heating provided using a hot-air blower (Leister). The sample temperature was monitored using a $0.5 \mathrm{~mm} K$-type thermocouple placed in the centre of the sample bed. The data treatment was performed using inhouse developed ProQEXAFS software (Clark et al., 2020). Calibration of the monochromator angle to energy of the incident X-ray beam was performed through measurement of an $\mathrm{Ni}$ foil. Prior to normalization, the data were filtered for high-frequency noise with a Butterworth filter and the application of a weak Savitzky-Golay moving time point smoothing (window size, five spectra). Edge-step normalization was performed using a first-order polynomial and Victoreen function in the pre-edge and post-edge regions after the timeframe averaging. The data were then interpolated onto an energy grid with $0.25 \mathrm{eV}$ steps in the XANES region and $0.025 \AA^{-1}$ steps in the EXAFS region. Ni speciation was performed through linear combination analysis of the treated spectra using reference spectra of $\mathrm{LaFe}_{0.8} \mathrm{Ni}_{0.2} \mathrm{O}_{3}, \mathrm{NiO}$ and metallic Ni. The Demeter software package (version 0.9.24) was used for linear combination fitting of the normalized spectra within the energy range $8.313-8.363 \mathrm{keV}$ (Ravel \& Newville, 2005).

\section{Acknowledgements}

The authors are grateful for the provision of beam time by the SLS. The work was conducted as part of the Swiss Competence Center for Energy Research (SCCER BIOSWEET) of the Swiss Innovation Agency (lnnosuisse).

\section{Funding information}

The Swiss National Science Foundation (SNF, No. 200021_159568) and the Competence Center for Energy and
Mobility (CCEM) are acknowledged for their financial support.

\section{References}

Abe, H., Aquilanti, G., Boada, R., Bunker, B., Glatzel, P., Nachtegaal, M. \& Pascarelli, S. (2018). J. Synchrotron Rad. 25, 972-980.

Błachucki, W., Szlachetko, J., Hoszowska, J., Dousse, J. C., Kayser, Y., Nachtegaal, M. \& Sá, J. (2014). Phys. Rev. Lett. 112, 173003.

Burnat, D., Kontic, R., Holzer, L., Steiger, P., Ferri, D. \& Heel, A. (2016). J. Mater. Chem. A, 4, 11939-11948.

Clark, A. H., Imbao, J., Frahm, R. \& Nachtegaal, M. (2020). J. Synchrotron Rad. 27, 551-557.

Fabbri, E., Nachtegaal, M., Binninger, T., Cheng, X., Kim, B. J., Durst, J., Bozza, F., Graule, T., Schäublin, R., Wiles, L., Pertoso, M., Danilovic, N., Ayers, K. E. \& Schmidt, T. J. (2017). Nat. Mater. 16, 925-931.

Fonda, E., Rochet, A., Ribbens, M., Barthe, L., Belin, S. \& Briois, V. (2012). J. Synchrotron Rad. 19, 417-424.

Frahm, R. (1988). Nucl. Instrum. Methods Phys. Res. A, 270, 578581.

Guda, A. A., Bugaev, A. L., Kopelent, R., Braglia, L., Soldatov, A. V., Nachtegaal, M., Safonova, O. V. \& Smolentsev, G. (2018). J. Synchrotron Rad. 25, 989-997.

Hansen, K., Reckleben, C., Diehl, I. \& Klär, H. (2008). Nucl. Instrum. Methods Phys. Res. A, 589, 250-258.

Jiang, S. P. (2008). J. Mater. Sci. 43, 6799-6833.

Lützenkirchen-Hecht, D., Grundmann, S. \& Frahm, R. (2001). J. Synchrotron Rad. 8, 6-9.

Marberger, A., Petrov, A. W., Steiger, P., Elsener, M., Kröcher, O., Nachtegaal, M. \& Ferri, D. (2018). Nat. Catal. 1, 221-227.

Marchionni, V., Szlachetko, J., Nachtegaal, M., Kambolis, A., Kröcher, O. \& Ferri, D. (2016). Phys. Chem. Chem. Phys. 18, $29268-29277$.

Marcilly, C., Courty, P. \& Delmon, B. (1970). J. Am. Ceram. Soc. 53, $56-57$.

Müller, O., Nachtegaal, M., Just, J., Lützenkirchen-Hecht, D. \& Frahm, R. (2016). J. Synchrotron Rad. 23, 260-266.

Müller, O., Stötzel, J., Lützenkirchen-Hecht, D. \& Frahm, R. (2013). J. Phys. Conf. Ser. 425, 092010.

Nachtegaal, M., Hartfelder, U. \& Van Bokhoven, J. A. (2017). Springer Series in Chemical Physics, Vol. 114, pp. 89-110. New York: Springer.

Nachtegaal, M., Müller, O., König, C. \& Frahm, R. (2015). X-ray Absorption and $X$-ray Emission Spectroscopy: Theory and Applications, Vols. 1-2, pp. 155-183. Chichester, UK: John Wiley \& Sons Ltd.

Nagai, Y., Dohmae, K., Ikeda, Y., Takagi, N., Tanabe, T., Hara, N., Guilera, G., Pascarelli, S., Newton, M. A., Kuno, O., Jiang, H., Shinjoh, H. \& Matsumoto, S. (2008). Angew. Chem. Int. Ed. 47, 9303-9306.

Neagu, D., Oh, T. S., Miller, D. N., Ménard, H., Bukhari, S. M., Gamble, S. R., Gorte, R. J., Vohs, J. M. \& Irvine, J. T. S. (2015). Nat. Commun. 6, 8120.

Nishihata, Y., Mizuki, J., Akao, T., Tanaka, H., Uenishi, M., Kimura, M., Okamoto, T. \& Hamada, N. (2002). Nature, 418, 164-167.

Onn, T. M., Monai, M., Dai, S., Fonda, E., Montini, T., Pan, X., Graham, G. W., Fornasiero, P. \& Gorte, R. J. (2018). J. Am. Chem. Soc. 140, 4841-4848.

Pascarelli, S., Neisius, T. \& De Panfilis, S. (1999). J. Synchrotron Rad. 6, 1044-1050.

Peña, M. A. \& Fierro, J. L. G. (2001). Chem. Rev. 101, 1981-2018.

Ravel, B. \& Newville, M. (2005). J. Synchrotron Rad. 12, 537-541.

Sapountzi, F. M., Gracia, J. M., Weststrate, C. J., Fredriksson, H. O. A. \& Niemantsverdriet, J. W. (2017). Prog. Energy Combust. Sci. 58, $1-35$. 
Smolentsev, G., van Vliet, K. M., Azzaroli, N., van Bokhoven, J. A., Brouwer, A. M., de Bruin, B., Nachtegaal, M. \& Tromp, M. (2018). Photochem. Photobiol. Sci. 17, 896-902.

Steiger, P., Delmelle, R., Foppiano, D., Holzer, L., Heel, A., Nachtegaal, M., Kröcher, O. \& Ferri, D. (2017). ChemSusChem, 10, 2505-2517.

Steiger, P., Nachtegaal, M., Kröcher, O. \& Ferri, D. (2018). ChemCatChem, 10, 4456-4464.
Szlachetko, J., Nachtegaal, M., de Boni, E., Willimann, M., Safonova, O., Sa, J., Smolentsev, G., Szlachetko, M., van Bokhoven, J. A., Dousse, J. C., Hoszowska, J., Kayser, Y., Jagodzinski, P., Bergamaschi, A., Schmitt, B., David, C. \& Lücke, A. (2012). Rev. Sci. Instrum. 83, 103105.

Wang, H., Yu, C., Wei, X., Gao, Z., Xu, G.-L., Sun, D.-R., Li, Z., Zhou, Y., Li, Q.-J., Zhang, B.-B., Xu, J.-Q., Wang, L., Zhang, Y., Tan, Y.-L. \& Tao, Y. (2017). J. Synchrotron Rad. 24, 667-673. 